\title{
Use of Caudal Analgesia Supplemented with Low Dose of Morphine in Children Who Undergo Renal Surgery
}

\author{
Boris Chertin ${ }^{\mathrm{a}} \quad$ Alexander Zeldin $^{\mathrm{b}} \quad$ Stanislav Kocherov $^{\mathrm{a}} \quad$ Alexander loscovich $^{\mathrm{b}}$ \\ Israel A. Ostrovsky ${ }^{b} \quad$ Yaacov Gozal ${ }^{b}$ \\ aDepartments of Pediatric Urology and ${ }^{b}$ Anesthesiology, Shaare Zedek Medical Center, Faculty of Medical Science, Hebrew University, \\ Jerusalem, Israel
}

\section{Key Words}

Caudal anesthesia $\cdot$ Pediatric • Renal surgery

\begin{abstract}
Introduction: To test the efficacy and safety of caudal anesthesia (CA) supplemented by low dose morphine in children who undergo renal surgery. Materials and Methods: Forty patients aged 2 months-14 years were enrolled and randomly divided into two groups of 20 patients each: Group A (bupivacaine 0.2\% with fentanyl); Group B (bupivacaine with morphine). The duration of surgery and hospitalization time were recorded. Postoperative pain score was measured by Face Legs Activity Cry Consolability scale and Wong-Baker Faces scale for those who are older. Overall use of rescue analgesics was calculated. Results: There was no statistical difference in the length of surgery, incidence of pruritus, postoperative nausea, vomiting and urinary retention between the two groups. However the postoperative opioid requirements were significantly higher in Group A $1.03 \pm 0.9 \mathrm{mg} / \mathrm{kg}$ compared to Group $B$, in which only one patient required opioid therapy ( $p<0.0001$ ). Moreover the need for non-opioid rescue analgesic was higher in Group A, $(36 \pm 5.7 \mathrm{mg} / \mathrm{kg}$ of paracetamol) compared to morphine CA group there only $26 \pm 3.6 \mathrm{mg} / \mathrm{kg}$ required during first 24 $h$ of the postoperative period $(p=0.0312)$. The Face Legs Ac-
\end{abstract}

\section{KARGER}

Fax +4161306 1234

E-Mail karger@karger.ch

www.karger.com
(C) 2015 S. Karger AG, Basel

$1015-9770 / 15 / 0093-0132 \$ 38.00 / 0$

Accessible online at:

www.karger.com/cur tivity Cry Consolability pain score (1, 4, and $24 \mathrm{~h}$ after surgery) and Wong-Baker Faces scale were significantly higher in Group A. The hospitalization period was shorter in the CA morphine group, but the difference did not reach statistical significance. None developed hemodynamic instability or respiratory depression. Conclusions: Our data show that CA supplemented with low dose morphine provides a longer duration of analgesia without significant side-effects in children undergoing renal surgery. Copyright $\odot 2015$ S. Karger AG, Basel

\section{Introduction}

Caudal analgesia (CA) with bupivacaine has gained popularity in the renal surgery providing painless postoperative period, earlier child feeding and a fast recovery $[1,2]$. However CA with bupivacaine provides painless postoperative period for a limited period of time and requires additional painkiller therapy [3-5]. CA supplemented with morphine or clonidine has demonstrated 
Table 1. Demographic data and surgical data $(\mathrm{N}=40)$

\begin{tabular}{lll}
\hline Data & Group 1 (N=20) & Group 2 (N=20) \\
\hline Age (range months-years) & $2-14$ & $1-14$ \\
Weight (kg) & $21 \pm 16$ & $22 \pm 13.8$ \\
Male & $8(40 \%)$ & $9(45 \%)$ \\
Female & $12(60 \%)$ & $11(55 \%)$ \\
ASA I & $19(95 \%)$ & $19(95 \%)$ \\
ASA II & $1(5 \%)$ & $1(5 \%)$ \\
Duration of surgery (min) & $94 \pm 27$ & $89 \pm 35$ \\
Type of surgery & & \\
Open pyeloplasty & 7 & 5 \\
Laparoscopic pyeloplasty & 4 & 6 \\
Laparoscopic vascular hitch & 2 & 3 \\
Laparoscopic nephrectomy & 6 & 5 \\
Laparoscopic partial nephrectomy & 1 & 1
\end{tabular}

$\mathrm{ASA}=$ American Society of Anesthesiologists

its benefits in children with regards to longer duration of analgesia and a decrease in supplemental postoperative opiates [6-10]. Unfortunately, these agents have potential side effects that can limit their use. The incidence of adverse effects is usually dose-dependent; therefore, it would be appropriate to use the lowest effective dose of these adjuncts $[6,11]$. Moreover the effect of the CA supplemented with either morphine or clonidine on patients undergoing renal surgery has not been reported yet in the clinical practice. Therefore we have designed this study in order to test the efficacy and safety of CA supplemented by low dose morphine in children who undergo renal surgery.

\section{Patients and Methods}

After obtaining institutional review board approval and signed parental informed consent, we have prospectively enrolled 40 children, aged 2 months-14 years, American Society of Anesthesiologists physical status I or II, who were scheduled to undergo renal surgery. No sedative premedication was given. The patients were monitored with electrocardiogram, non-invasive blood pressure, pulse oximetry, temperature, capnography, and end tidal anesthetic concentration.

Intravenous induction for general anesthesia with endotracheal intubation is commenced utilizing intravenous propofol $3 \mathrm{mg} / \mathrm{kg}$, atracurium $0.5 \mathrm{mg} / \mathrm{kg}$, without intravenous opioids.

After the induction of general anesthesia, the patients were randomized into $2 \mathrm{CA}$ groups of 20 patients each: Group A (1.2 $\mathrm{ml} / \mathrm{kg}$ of bupivacaine $0.2 \%$ with fentanyl $2 \mu \mathrm{g} / \mathrm{kg}$ ); Group B (1.2 $\mathrm{ml} / \mathrm{kg}$ of bupivacaine $0.2 \%$ with morphine $15-20 \mu \mathrm{g} / \mathrm{kg}$ ). In order to ensure a balance in the sample size across groups over time we have used a block type of the randomization.

Caudal Anesthesia Supplemented with

Low Dose of Morphine

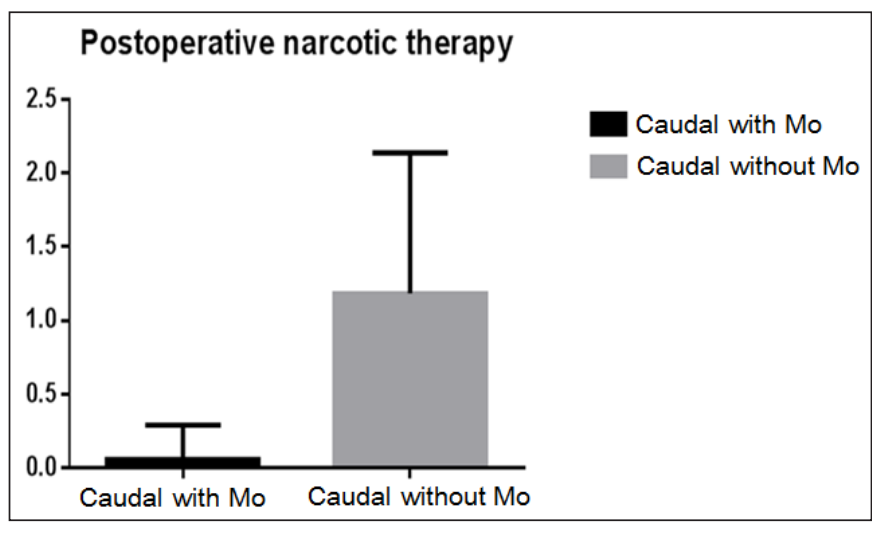

Fig. 1. Postoperative narcotic therapy.

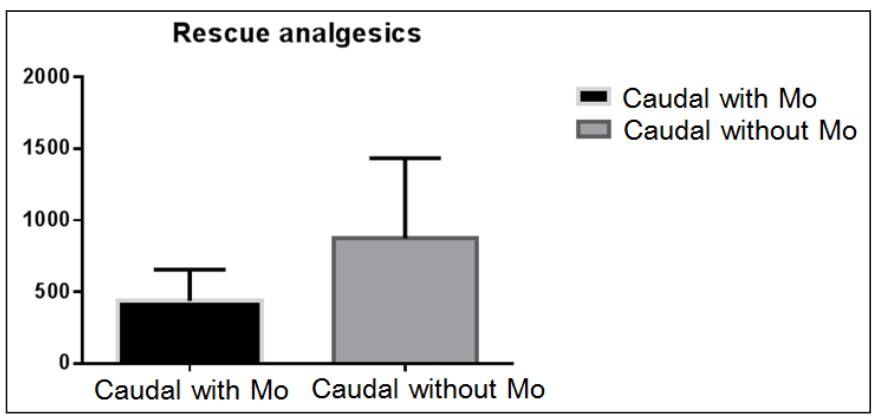

Fig. 2. Rescue analgesics.

The child was then turned to the lateral position, and caudal blockade was performed using a 21 or 23-gauge needle connected to the $10 \mathrm{ml}$ syringe inserted through the sacrococcygeal ligament into the caudal space by the same anesthesiologist. After negative aspiration for blood or cerebrospinal fluid the study solution was administered over $1 \mathrm{~min}$. Anesthesia was maintained using controlled ventilation with sevoflurane in $50 \% \mathrm{~N}_{2} \mathrm{O} / 50 \% \mathrm{O}_{2}$. The inhaled concentration of sevoflurane was adjusted to maintain hemodynamic stability, which was defined as a change in systolic blood pressure and heart rate of no more than $20 \%$ of baseline parameters. At the commencement of surgery all patients had received intravenous dexamethasone $0.2 \mathrm{mg} / \mathrm{kg}$ and at the end ondansetron $0.1 \mathrm{mg} / \mathrm{kg}$. The operation was performed without using i.v. fentanyl, or other opioids or sedative drugs.

Mean arterial pressure, heart rate, and maintenance end-tidal sevoflurane concentration were registered every $5 \mathrm{~min}$. The patient was extubated based on clinical criteria. The patient was then transported to the post-operative anesthesia care unit. The duration of surgery and hospitalization time were recorded. Postoperative pain score was measured by pediatric observational face, legs, activity, cry, consolability (FLACC) scale (0-10 score range) FLACC scale for those who were younger than 3 years old, and

Curr Urol 2015;9:132-137 


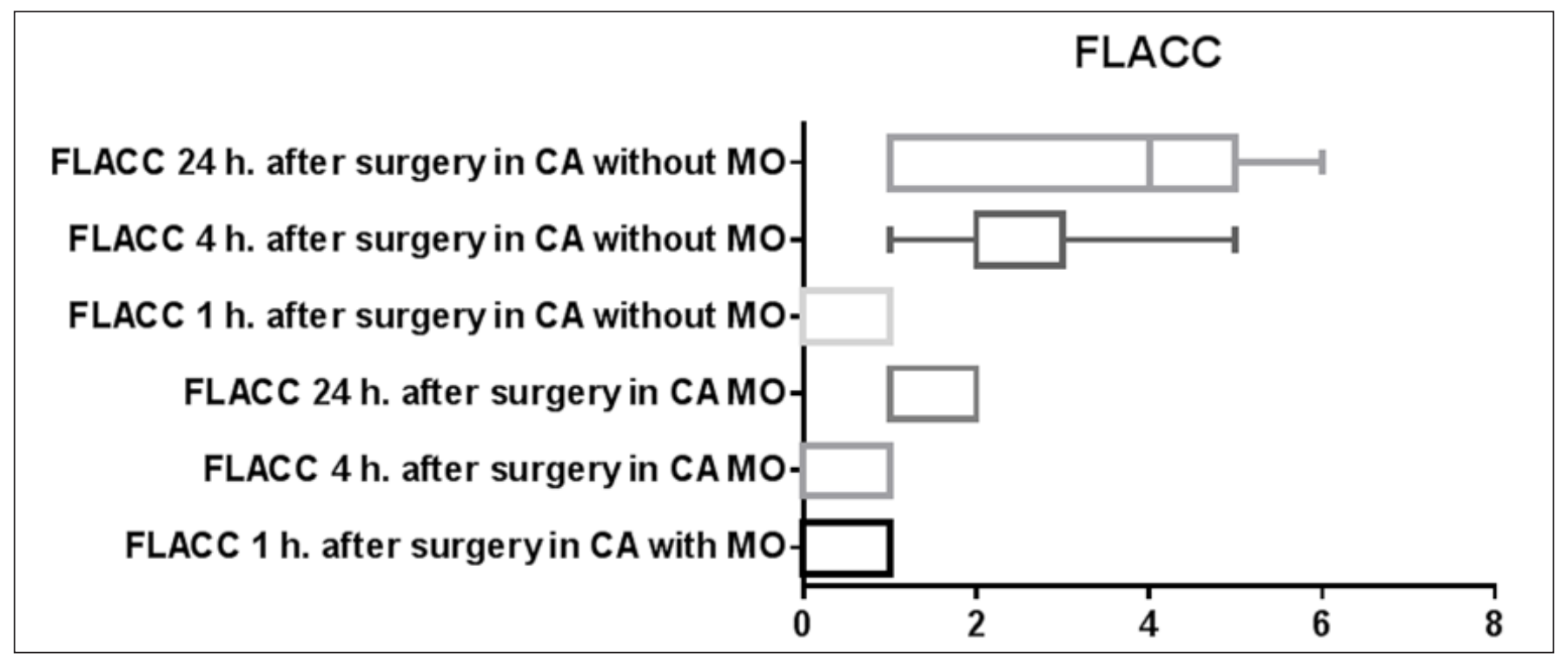

Fig. 3. FLACC

Wong-Baker Faces (WBF) scale for those were older 1, 4 and 24 hour after surgery. The intensity of pain utilizing FLACC was classified as none or mild $(\leq 4)$, moderate $(5-7)$, and severe $(\geq 8)$. Overall use of rescue analgesics was calculated. The leg component of the FLACC was not counted and taking into consideration one hour after surgery cessation due to possible motor block after CA.

Similarly to other authors, we routinely start analgesic treatment with weak analgesics and reserve opioids for more intense pain $[6,12,13]$. Paracetamol is an analgesic drug used in our country for the treatment of postoperative pain in children and it is also our routine to use it as the first analgesic rescue agent. If necessary, we also use other analgesic drugs (NSAIDs and opioids). Thus, in the present study, postoperative pain was treated firstly with paracetamol, followed by ibuprofen or morphine according to the intensity of the pain. If the patient presented with pain between the recorded intervals of FLACC or WBF another evaluation was done in order to determine the intensity of the pain and the type of analgesic to use. The postoperative analgesia time (time from the caudal anesthesia to the first use of analgesics) and the number of doses of rescue analgesics (paracetamol, ibuprofen, or morphine) in the postoperative period $(24 \mathrm{~h})$ were recorded. Mean arterial pressure, heart rate, and respiratory rate were monitored during the first $24 \mathrm{~h}$ of the postoperative period. Postoperative nausea and vomiting was recorded in the same periods as those used for pain assessment. Rescue treatment of postoperative nausea and vomiting if needed was done with ondansetron $(0.1$ $\mathrm{mg} / \mathrm{kg}$ ) given at intervals of $4 \mathrm{~h}$. Side effects such as urinary retention and pruritus were also noted.

An a priori study sample size calculation was performed based upon assumed $60 \%$ difference in the pain status between Group A and B. Applying an uncorrected $\chi^{2}$ test with an $\alpha$ of 0.05 , a sample size of 20 would be expected to have an $80 \%$ power to detect a minimum $60 \%$ difference between two such treatment groups.
Commercially available software GraphPad Prism version 6.02 for Windows, (GraphPad software, San Diego, CA) was used. Mann-Whitney and Fisher test were utilized for statistical evaluation, considering $\mathrm{p}$ value of $<0.05$ as significant.

\section{Results}

There was no difference with regards to the type and length of renal surgery, weight, sex and age of the patients between the two groups (table 1). There was no statistical difference in the incidence of pruritus, postoperative nausea, vomiting and urinary retention between the two groups. However the analgesia time was significantly shorter in the first group $320 \pm 56$ min (mean \pm $\mathrm{SD})$ compared to the second group there analgesia time was $410 \pm 32 \mathrm{~min}(\mathrm{p}=0.018)$. Furthermore the postoperative opioid requirements were significantly higher in the first group $1.03 \pm 0.9 \mathrm{mg} / \mathrm{kg}$ compared to the second group in which only one patient required opioid therapy $(\mathrm{p}<0.0001)$ (fig. 1). Moreover the need for non opioid rescue analgesic was higher in the first group, (36 \pm 5.7 $\mathrm{mg} / \mathrm{kg}$ of paracetamol) compared to morphine CA group there only $26 \pm 3.6 \mathrm{mg} / \mathrm{kg}$ required during first $24 \mathrm{~h}$ of the postoperative period ( $\mathrm{p}=0.0312$ ) (fig. 2$)$. The FLACC pain score $(1,4$, and $24 \mathrm{~h}$ after surgery) and WBF scale were significantly higher in the Group A fig. 3 and fig. 4 respectively. The hospitalization period was shorter in 


\section{Wong-Baker Faces (WBF) scale}

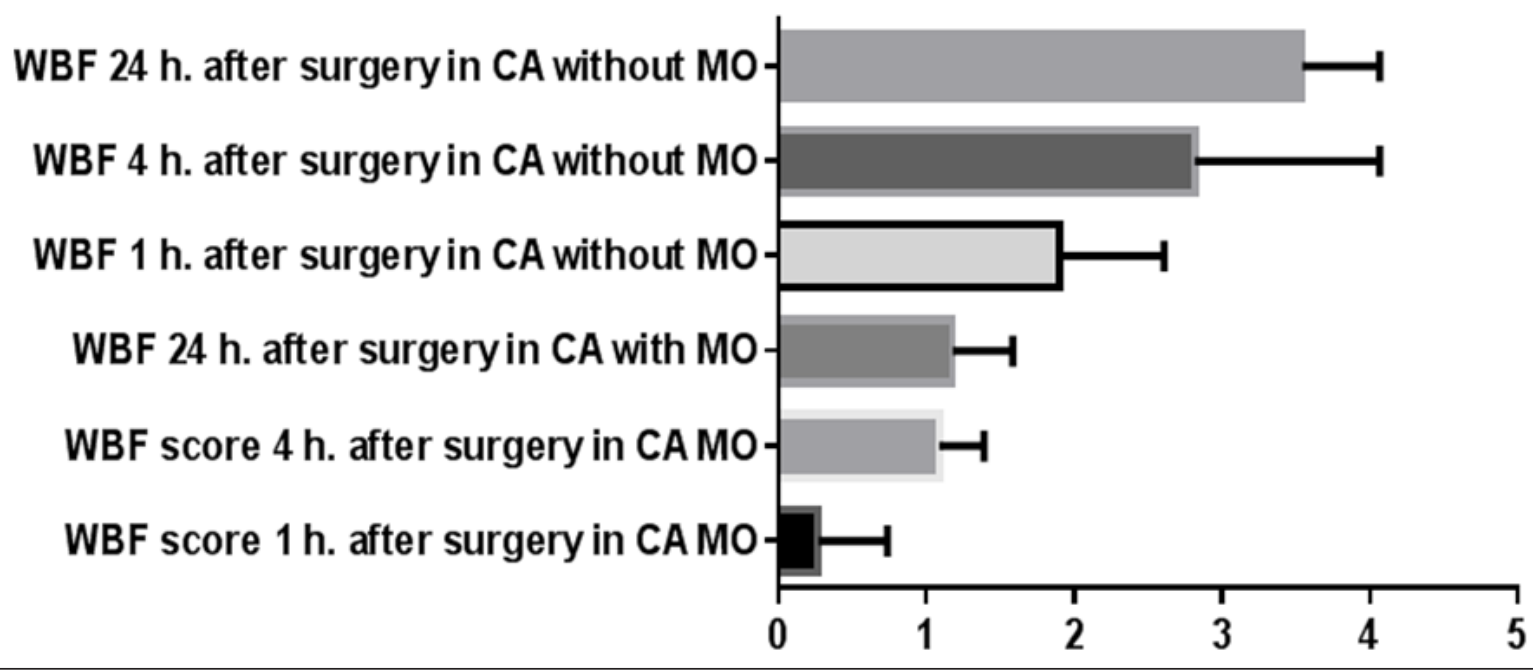

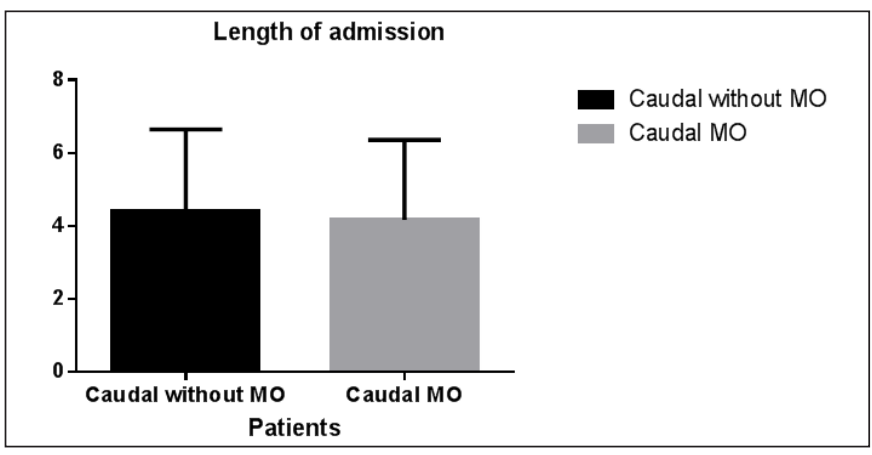

Fig. 5. Length of admission.

the CA morphine group, but the difference did not reach statistical significance (fig. 5). None developed hemodynamic instability or respiratory depression.

\section{Discussion}

Caudal block is a most popular pediatric regional anesthesia. Its first was described in 1933 and has gained a big popularity in the early $60 \mathrm{~s}[1,2]$. There are a lot of advantages of this technique. It is easy to teach and only 30 caudal blocks are required for pediatric anesthesiol-

Caudal Anesthesia Supplemented with Low Dose of Morphine
Fig. 4. Wong-Baker Faces scale

ogy resident or fellow to reach the same level as a senior doctor. It can be used in the outpatient surgery providing an adequate level of preoperative analgesia without delay in the hospital discharge. It can be used in the different operative setups from infraumbilical or genital to the open heart surgery [2, 6-8]. However some drawbacks of this procedure cannot be brushed aside. It is suggested to be used only as a single shot technique due to possible catheters contamination from fecal soiling. In order to obtain a more cephalic spread only high volume of the anesthetic mixture should be used. Furthermore different degree complications of this technique from purities and nausea to the respiratory arrest and even paraplegia were reported [2].

Plain bupivacaine, levobupivacaine and ropivacaine are widely utilized in caudal block [5,8]. It has been proved by the several authors that metameric spread of these analgetics depends on the volume of injected mixture while the desired density of the block (less dense for postoperative more dense for intraoperative analgesia) depends on the concentration of anesthetic. The most frequent method to prolong a postoperative anesthesia is to add different adjunct drugs to the local anesthetic solution. These additives can be divided in non-opioids (clonidine, ketamine) and opioids (morphine, fentanyl) $[1,4,8,10,11]$. The use of caudal opioids significantly prolongs the duration of analgesia but may carry a num-

Curr Urol 2015;9:132-137 
ber of unpleasant side effects such as nausea, vomiting, pruritus or urinary retention and even a risk of later respiratory depression [6]. For these reasons the utilization of the opioids in caudal pediatric block is underuse.

Most studies describe the efficacy of caudal anesthesia for infraumbilical surgery $[6,10,11]$. We have not found any study regarding the use of caudal anesthesia in supraumbilical abdominal or renal surgery. Furthermore even the recent survey among the members of the Society for Pediatric Urology regarding their preferences for intraoperative anesthesia and postoperative pain control has referred to the use of epidural/caudal anesthesia as the same type of the anesthesia [15]. The efficacy and advisability of continuous epidural anesthesia were evaluated in patients following open dismembered pyeloplasty. Ben-Meir et al. [16] demonstrated that the use of the epidural anesthesia in these patients did not show any benefits in terms of need in rescue analgesia, mobilization and discharge time compared with patients on nonepidural analgesia protocol. The important finding of their study was that each group of studied patients demonstrated $20 \%$ of side effects. The most common side effects were nausea, vomiting, itching and some patients from the epidural group presented with motor block.

Our data showed that caudal anesthesia supplemented with low dose of morphine was beneficial in the postoperative pain control compared with patients who received a routine caudal block. The patients from caudal morphine group required significantly less rescue non and opioid analgesia, the time for the discharge home was also shorten in this group although the difference did not reach statistical significance. The earlier publications regarding the use of caudal morphine in various surgical procedures justified $0.03 \mathrm{mg} / \mathrm{kg}$ of morphine as an initial dosage to achieve a good level of the postoperative analgesia [6]. However, the later publications showed that even $0.01 \mathrm{mg} / \mathrm{kg}$ may provide a reasonable level of postoperative pain control following minor outpatient surgery [12]. Although these publications have justified low dosages of the used morphine the same authors continued to emphasize that up to $23 \%$ of the patients with CA morphine experienced nausea and vomiting during postoperative period, $3 \%$ complained on voiding disturbances required catheter insertion, and up to $10 \%$ suffered of pruritus. The interesting finding in our study was that none of our patients in morphine group suffered of vomiting, pruritus or urinary retention and only one patient complained of nausea during the first 12 hours after surgery. We have related our level of the side effects to low dosage of morphine used in our patients on the first place. Children who weighed less that $10 \mathrm{~kg}$ received 15 $\mu \mathrm{g} / \mathrm{kg}$ and those who weighed more than $10 \mathrm{~kg}$ received $20 \mu \mathrm{g} / \mathrm{kg}$ of morphine. Moreover as we have aforementioned all patients at the commencement of surgery had received intravenous dexamethasone $0.2 \mathrm{mg} / \mathrm{kg}$ and at the end of surgery ondansetron $0.1 \mathrm{mg} / \mathrm{kg}$ thus none presented with gastrointestinal bothering related to the CA morphine analgesia. Furthermore none of our patients reported voiding problems related to the caudal block following procedure and none experienced respiratory depression.

In our series efficacy was evaluated based on FLACC and Visual Analog Scale for Pain scores. The FLACC has been tested, and its validity and interpreter reliability were established in 1997 [17]. Von Baeyer et al. [18] found that the FLACC has moderate concurrent validity with the Faces Pain Scale and good validity with Visual Analog Scale for Pain. They noted that the FLACC is highly recommended for use in studies since it has excellent evidence of reliability, validity and responsiveness. It cannot be assumed that the 0 to 10 scale of the FLACC is psychometrically equivalent to an ideal or self-reported 0 to 10 scale since scores representing mild, moderate and severe pain, minimally clinically significant difference and the equality of intervals between scores are not well established. However, the 2 scales are meant to interpret pain scores as low, moderate or high in severity. In view of these and many more publications, and given that there is no single tool that is valid for all pediatric ages, we combined the mild, moderate and severe score groups according to the 2 tools used.

This manuscript is not without limitations, which are worth to be mentioned. The main limitations of the study are non-blinded randomization and observer bias. Nurses and parents could not be blinded to the treatment arm due to the hospital regulations. We presented here relatively small cohorts of patients; therefore we did not reach a statistical significance in some evaluated parameters between two groups. We have included patients who underwent different types of renal surgery; however a main aim of this study was to demonstrated efficacy and safety of supplemented by morphine caudal surgery in patients undergo renal surgery. The inclusion of patients who underwent laparoscopic pyeloplasty may explain relatively long stay of some patients after surgery. It is our routine practice to keep bladder catheter indwelling in those patients who have an anasthomotic urinary leak till its cessation. However, including heterogeneous patients allowed us to check out the benefits of this type of regional 
anesthesia in the different clinical set ups. Finally in order to evaluate respiratory status of patients following caudal block with morphine and to define a safety of this procedure in every clinical setup including ambulatory surgery end tidal $\mathrm{CO}_{2}$ levels should be measured in these patients during first 24 hours after surgery. This study is currently undergoing in our department.

\section{Conclusion}

Our data show that CA supplemented with low dose morphine provides a longer duration of analgesia without significant side-effects in children undergoing renal surgery.

\section{References}

1 Krane EJ, Tyler DC, Jacobson LE: The dose response of caudal morphine in children. Anesthesiology 1989;71:48-52.

2 Chalkiadis G: The rise and fall of continuous epidural infusions in children. Paediatr Anaesth 2003;13:91-94.

3 Rosen KR, Rosen DA: Caudal epidural morphine for control of pain following open heart surgery in children. Anesthesiology 1989;70: 418-421.

4 Valley RD, Bailey AG: Caudal morphine for postoperative analgesia in infants and children: a report of 138 cases. Anesth Analg 1991;72:120-124.

5 Thies KC, Driessen J, Kho HG, Kwak K, Knoll J, de Gier R, Feitz W: Longer than expected duration of caudal analgesia with two different doses of levobupivacaine in children undergoing hypospadias repair. J Pediatr Urol 2010;6:585-588.

6 Mayhew JF, Brodsky RC, Blakey D, Petersen W: Low-dose caudal morphine for postoperative analgesia in infants and children: a report of 500 cases. J Clin Anesth 1995;7:640-642.

7 Leong CK, Ng AS, Chew SL: Caudal morphine in paediatric patients: a comparison of two different doses in children after major urogenital surgery. Ann Acad Med Singapore 1998;27:371-375.
8 Vetter TR, Carvallo D, Johnson JL, Mazurek MS, Presson RG Jr: A comparison of single-dose caudal clonidine, morphine, or hydromorphone combined with ropivacaine in pediatric patients undergoing ureteral reimplantation. Anesth Analg 2007;104:13561363.

9 Singh R, Kumar N, Singh P: Randomized controlled trial comparing morphine or clonidine with bupivacaine for caudal analgesia in children undergoing upper abdominal surgery. Br J Anaesth 2011;106:96-100.

10 Fernandes ML, Pires KC, Tibúrcio MA, Gomez RS: Caudal bupivacaine supplemented with morphine or clonidine, or supplemented with morphine plus clonidine in children undergoing infra-umbilical urological and genital procedures: a prospective, randomized and double-blind study. J Anesth 2012;26: 213-218.

11 El-Hennawy AM, Abd-Elwahab AM, Abd-Elmaksoud AM, El-Ozairy HS, Boulis SR: Addition of clonidine or dexmedetomidine to bupivacaine prolongs caudal analgesia in children. Br J Anaesth 2009;103:268-274.

12 Cesur M, Alici HA, Erdem AF, Yapanoglu T, Silbir F: Effects of reduction of the caudal morphine dose in paediatric circumcision on quality of postoperative analgesia and morphine-related side-effects. Anaesth Intensive Care 2007;35:743-747.
13 Merguerian PA, Sutters KA, Tang E, Kaji D, Chang B: Efficacy of continuous epidural analgesia versus single dose caudal analgesia in children after intravesical ureteroneocystostomy. J Urol 2004;172:1621-1625.

14 Stewart DW, Ragg PG, Sheppard S, Chalkiadis GA: The severity and duration of postoperative pain and analgesia requirements in children after tonsillectomy, orchiopexy, or inguinal hernia repair. Paediatr Anaesth 2012;22:136-143.

15 Morrison K, Herbst K, Corbett S, Herndon $\mathrm{CD}$ : Pain management practice patterns for common pediatric urology procedures. Urology 2014;83:206-210.

16 Ben-Meir D, Livne P M, Katz J, Gelman O, Efrat R: Continuous epidural versus nonepidural analgesia for post-pyeloplasty pain in children. J Urol 2009;182(4 suppl):1841-1844.

17 Merkel SI, Voepel-Lewis T, Shayevitz JR, Malviya S: The FLACC: a behavioral scale for scoring postoperative pain in young children. Pediatr Nurs 1997;23:293-297.

18 von Baeyer CL, Soagryd LJ: Systematic review of observational (behavioural) measures of pain for children and adolescents age 3 to 18 years. Pain 2007;127:140-150. 